\title{
Thoracic endovascular aortic repair: The basics
}

\author{
Nick Cheshire, MD, FRCS, and Colin Bicknell, MD, FRCS
}

\begin{abstract}
Endografting for the treatment of thoracic aortic pathology continues to gain popularity; in some countries, numbers of endovascular aortic repairs now exceed those of open surgical cases. The skills and understanding of open surgical teams are not always translated into endovascular intervention teams, which may be led by cardiologists or vascular surgeons with little knowledge of thoracic pathology. Our experience with more than 400 thoracic interventional cases leads us to believe that that the cardiovascular surgeon is crucial to the success of any thoracic endovascular aortic repair program. Experienced surgeons should be involved in preoperative planning of cases, assessment of access vessels, creation of landing zones with revascularization procedures, passage of stents through the thoracic aorta, and protection of the spinal cord. In addition, surgeons should be familiar with the most common complications of thoracic endovascular aortic repair and be able to use both open surgical and endovascular strategies for complication management. (J Thorac Cardiovasc Surg 2013;145:S149-53)
\end{abstract}

Endografting to treat disease of the thoracic aorta was first described by Dake and colleagues ${ }^{1}$ almost 20 years ago. Since that time, a number of publications have demonstrated the safety of endografting in the thoracic aorta. ${ }^{2-4}$ In the developed world, the number of thoracic stent-grafts being placed is rising rapidly, and in some countries it now exceeds the number of open procedures being performed on the thoracic aorta. ${ }^{5}$ Coincidentally, the management of thoracic aortic disease may be moving from its traditional base in cardiovascular surgery to clinicians with less experience in open thoracic procedures, such as cardiologists and peripheral vascular surgeons. Our 15 -year experience with more than 400 thoracic endografts leads us to believe that the cardiovascular surgeon is a vital component in a thoracic endovascular aortic repair (TEVAR) team and should bring his or her understanding of the thoracic disease to all stages, from planning through TEVAR placement, and to adjunct techniques from protection of the spinal cord to specific postoperative care. In addition, we believe that all surgeons involved in TEVAR should be aware of the common complications and be able to manage both open surgical and endovascular strategies for correction.

The term TEVAR in this article will be used to include placement of an endovascular stent to treat any of the common thoracic aortic pathologies (aneurysm, dissection, penetrating ulcer, trauma, etc). TEVAR is most often an aortoaortic tube graft procedure and therefore should be more technically

\footnotetext{
From the Department of Surgery and Cancer, Imperial College School of Medicine, London, UK; and Imperial Vascular Unit, Imperial College Healthcare NHS Trust, London, UK.

Disclosures: Drs Cheshire and Bicknell have nothing to disclose with regard to commercial support.

Read at The American Association for Thoracic Surgery Aortic Symposium, New York, New York, April 26-27, 2012.

Received for publication July 5, 2012; revisions received Aug 12, 2012; accepted for publication Nov 28, 2012

Address for reprints: Nick Cheshire, MD, FRCS, Imperial College London, St Marys Hospital, London W2 1NY (E-mail: nick.cheshire@imperial.ac.uk). $0022-5223 / \$ 36.00$

Copyright (c) 2013 Published by Elsevier Inc. on behalf of The American Association for Thoracic Surgery

http://dx.doi.org/10.1016/j.jtcvs.2012.11.069
}

straightforward than endovascular aneurysm repair for abdominal aortic disease, which usually requires an aortoiliac bifurcated system. In practice, however, decision making before and during TEVAR is usually more complex than for endovascular aneurysm repair for abdominal aortic disease. This is because of the wide range of pathologies that may be seen in the thorax, the potential impact of covering supra-aortic branches, difficulty with access through the iliofemoral arteries, tortuosity in the thoracic aorta itself (Figure 1), and the nature of patients with thoracic aortic disease.

\section{PLANNING FOR TEVAR}

In our practice, high-resolution computed tomographic (CT) scanning is the primary investigative tool used for preoperative planning and TEVAR preparation. Other crosssectional modalities, such as magnetic resonance imaging, may be used; in our experience, however, the CT scan image allows easy interpretation by most members of the surgical and radiology team. This in turn makes planning discussions transparent and understandable. CT imaging should always extend from the supra-aortic vessels to the common femoral arteries. Multiplanar reconstructions of the data set are needed for the best assessment of the shape of the diseased thoracic segment and to provide details of proximal and distal landing zones (Figure 1). A variety of planning-specific software packages are available; these usually import the CT data and use preset parameters to reconstruct images automatically and make measurements easier. In our experience, this type of system is useful for highly complex endografts, such as fenestrated or branched devices, but is rarely needed for straightforward TEVAR. In many of our cases, the proprietary software supplied with a contemporary CT scanner is sufficient for accurate imaging and planning of TEVAR interventions.

Highly angulated segments of the aorta can be particularly challenging (Figure 1). Although there have been significant improvements in delivery device design by most if not all stent-graft manufacturers, knowledge of device-specific 


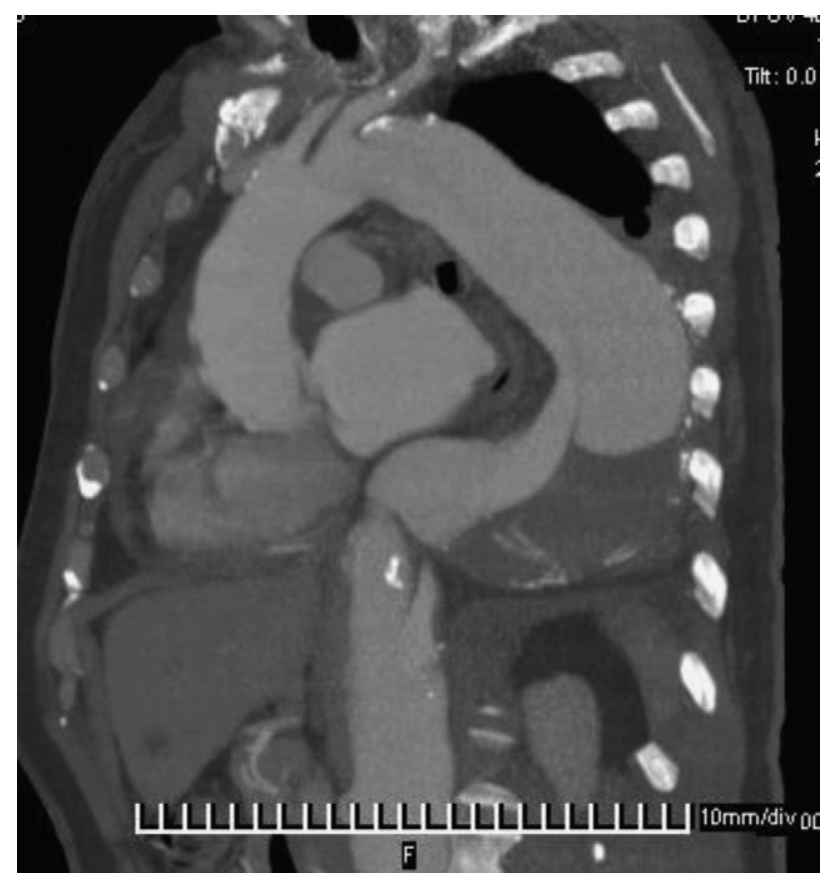

FIGURE 1. Multiplanar reconstruction of computed tomographic data showing tortuous anatomy in the thoracic aorta distal to a postdissection aneurysm. This kind of problem can be difficult to negotiate with a large-caliber stent-graft delivery system. Knowledge of individual thoracic endovascular aortic repair device characteristics, as well as adjuncts such as dual ("buddy") stiff wire passage and large sheath use, can be invaluable in overcoming such problems. Rupture is always a possibility.

characteristics or use of delivery sheaths is often a necessary requirement for the positioning of stent-grafts. Consideration of placement from above (through axillary or subclavian artery access) may occasionally be needed.

To assess the iliac arteries for access, a combination of multiplanar reconstruction and cross-sectional imaging is best in our experience. On cross-sectioning, particular attention should be paid to the diameter of the external iliac arteries (EIAs). Healthy, noncalcified vessels can usually easily accommodate a delivery system $1 \mathrm{~mm}$ larger in diameter than the vessel size (eg, a noncalcified 6-mm external iliac artery will usually allow passage of a $21 \mathrm{~F}[7 \mathrm{~mm}]$ system). Heavily calcified external iliac arteries, or those with significant focal stenosis, will only allow device passage if their native diameter is significantly larger than the device size. Great care should be exercised when planning TEVAR in female patients with iliac artery disease; this is a group in which injury can commonly occur.

Focal iliac stenosis can be considered for preoperative angioplasty. This is most valuable in common iliac disease. The accepted wisdom is to balloon dilate the vessel first, followed by stent-graft passage. If an iliac stent is required, this should be placed after the stent-graft procedure. In our series, fewer than $2 \%$ of patients have undergone preintervention angioplasty, with a low rate of iliac injury.

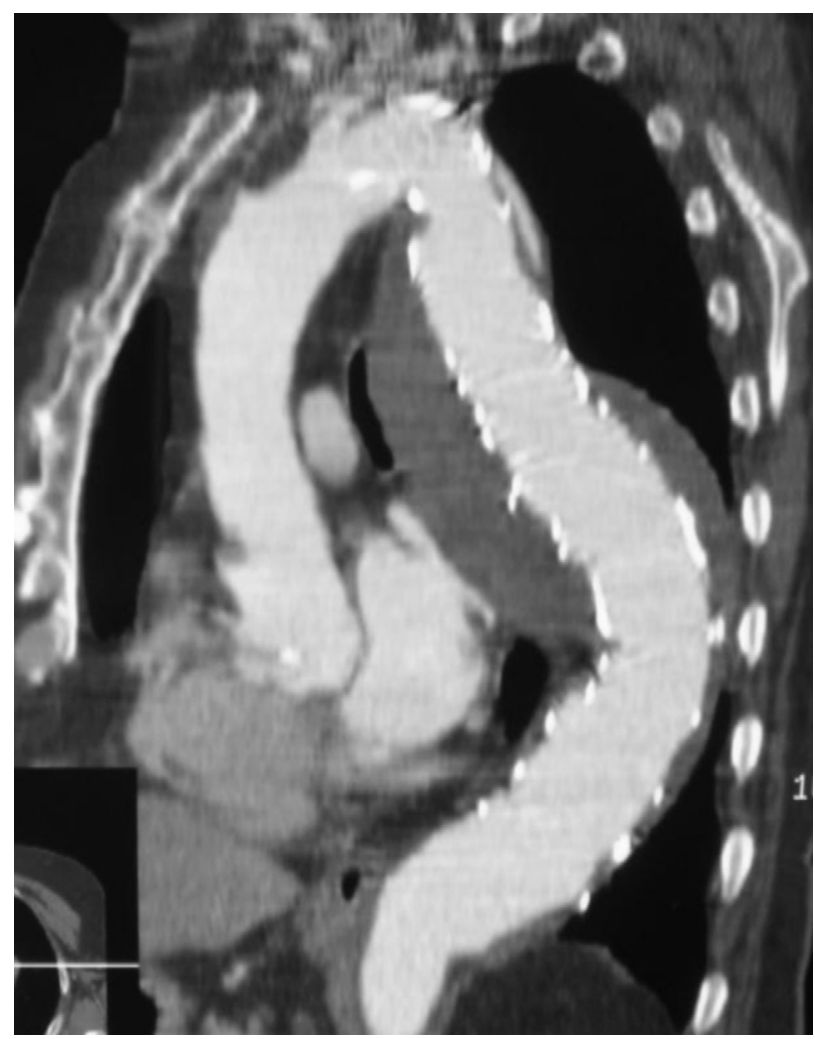

FIGURE 2. High angulation at the proximal landing zone in a case of thoracic aneurysm. In this case, the shape of the aortic arch dictates the landing zone. A minimum of $10 \mathrm{~mm}$ of relatively straight, nonaneurysmal aortic wall with limited mural thrombus is required for sealing with most devices. Angulation requires relatively greater length to secure a seal. The device in this case can be seen to be sitting away from the inferior arch wall, reducing the length of the seal.

Multiplanar reconstructions of the iliac arteries will best demonstrate the degree of tortuosity. We find iliac convolutions to pose less of an access problem than stenosis or calcification; commonly the iliac system, when not overly diseased, will straighten out when a stiff guide wire is passed through. Highly angulated iliac vessels $\left(90^{\circ}\right.$ or more) or those with several angles can be more difficult to pass through. This is particularly relevant when orientation of a fenestrated, branched, or scalloped device is used.

In patients with disease limited to the descending aorta, $10 \mathrm{~mm}$ of healthy proximal and distal aortic landing zonewith parallel walls and minimal adherent thrombus-will usually suffice for a seal with the commonly available stent-graft systems and will rarely migrate with active fixation (barbs, hooks, or pins). It is important that the segment does not contain an angle greater than $45^{\circ}$ if a $10-\mathrm{mm}$ landing zone is to be accepted. Steep angles, adherent mural thrombus, or both require respectively greater lengths as a sufficient sealing zone. This is particularly relevant at the proximal end when the disease is close to the aortic arch. Highly angulated arch shapes are common with large aneurysms (Figure 2). 


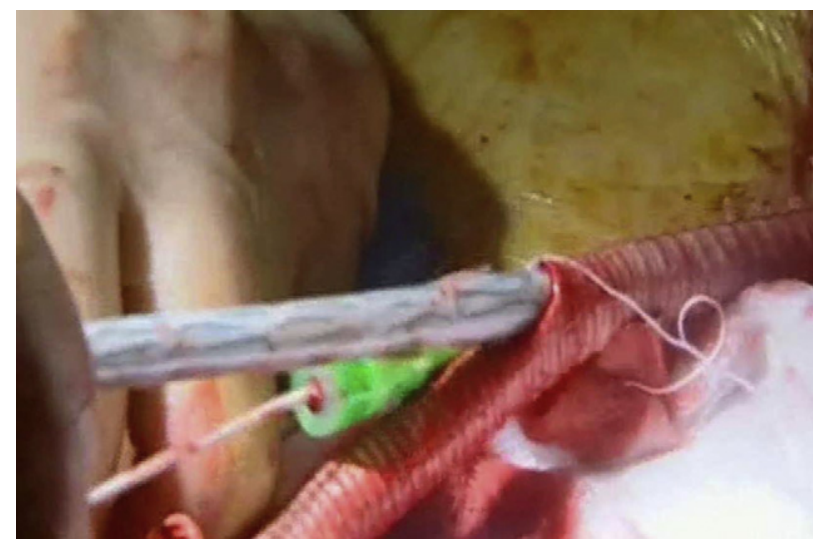

FIGURE 3. Use of a 10-mm diameter Dacron polyester fabric access conduit in the case of a small-caliber, calcified external iliac artery in a female patient. The conduit was sutured end to side to the common iliac artery, accessed through a muscle-splitting incision in the iliac fossa, and brought out in a straight line through a stab incision in the body wall. It is simplest to clamp the end of the graft and use needle access through the Dacron fabric to maintain hemostasis.

\section{OVERCOMING ILIAC ACCESS PROBLEMS}

There are a number of advanced endovascular techniques that can be used to overcome heavily diseased and calcified EIAs. These include multiple sites of angioplasty, either directly to the vessel or from within a covered stent-graft (so-called "cracking and paving" technique ${ }^{6}$ ).

We have used many of these techniques in our series, but we preferentially use an iliac surgical conduit. This is relatively easy to perform. Although the length of stay of patients may be increased using this technique, the rates of iliac injury are reduced significantly. ${ }^{7}$ We prefer an oblique, muscle-splitting incision in the appropriate iliac fossa followed by blunt extraperitoneal dissection to expose the iliac arteries. We prefer end-to-side anastomosis of a 10-mm Dacron polyester fabric graft onto the common iliac artery, with the prosthesis brought out through a stab incision in the body wall. We have found that clamping the end of the graft and puncturing it de novo (Figure 3) is the most efficient method to control direct bleeding from access through an iliac conduit. At the end of the procedure, we oversew a short stump of the conduit unless we have concerns about infection. In cases where there is sufficient concern that reintervention will be required, it may be useful to anastomose the distal end to the common femoral artery (as an iliofemoral bypass), which can later be used for access through the groin. In our practice, however, this is rarely used.

\section{COMPLICATIONS OF ILIAC ARTERY ACCESS Rupture}

When preoperative imaging suggests small or complex EIA access, or when intraoperative findings suggest iliac

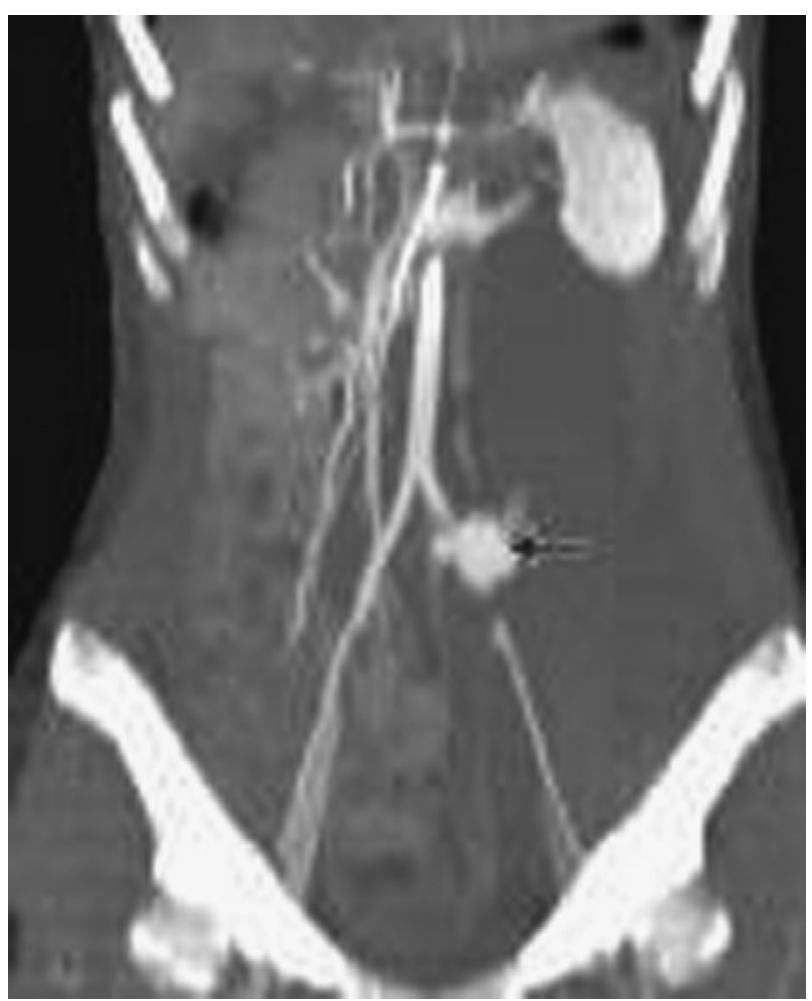

FIGURE 4. Angiogram showing extravasation of contrast from iliac artery rupture. This complication should be suspected whenever smallcaliber iliac arteries have been cannulated or when intraoperative device passage difficulties are encountered. On-table angiography before removal of the delivery system can be used for diagnosis. Never remove the device until control has been achieved, usually with an occlusion balloon passed into the lower aorta from the contralateral femoral artery.

difficulty, it is important to maintain a high level of suspicion of iliac artery injury. Every surgeon who has been involved in significant numbers of TEVAR procedures has seen the iliac artery, usually as the device is removed at the end of the procedure, bringing a length of the EIA with it.

When iliac artery injury is suspected, the key is early detection and prevention of hemorrhage. Before removing the large caliber-delivery sheath and stiff wire-but with the device withdrawn into the very distal EIA — angiography should be performed to look for extravasation (Figure 4).

If the EIA has been ruptured, the device must not be removed. Control of distal iliac flow can be obtained by placement of a large-caliber occlusion balloon through the opposite femoral artery. This balloon can be used to occlude the distal aorta before the ipsilateral delivery system is removed. With sufficient endovascular experience, a covered endograft can often be used to repair the disruption. It should be standard practice to include aortic occlusion balloons and covered stent-grafts for iliac repair in the emergency equipment stock for TEVAR. Alternatively, if devices or experience are not available, a surgical incision 
can be made in the iliac fossa, and open repair can then be undertaken in a relatively blood-free field.

\section{Occlusion}

Large-caliber delivery systems passing through the iliac system can result in lower limb ischemia, most usually as a consequence of iliac dissection. Suspicion should arise during removal of the endovascular system if there is not vigorous flow into the surgical field in the groin. As with rupture, a high level of suspicion appropriate to thorough knowledge of the preoperative anatomy and intraoperative problems should be maintained. Whenever a problem is suspected, a key point is not to lose the position of the wire across the arterial segment until the diagnosis has been confirmed.

Occlusion of the iliac artery, or of the common femoral artery, can be dealt with by either endovascular or open surgical means. For iliac dissection-in the common iliac artery or the EIA - a bare metal stent can usually be used to pin back the dissection flap and maintain flow. More rarely, open surgical repair may be needed.

In the common femoral artery, patch repair of the arteriotomy, with or without endarterectomy, is usually sufficient to restore normal flow into the leg. Sometimes this may be supplemented by embolectomy with balloon catheters with or without angiographic imaging.

Rarely, in prolonged procedures with occlusion of the arterial supply of the lower limb for longer than 6 hours (usually during complex fenestrated or branched stentgraft procedures dogged by complications), late ischemia may ensue after reperfusion, with compartment syndrome. In such cases, fasciotomy incisions are mandatory.

\section{Bleeding}

After large-caliber access for TEVAR, the clinical team must remain aware of the risk of groin vessel bleeding. This can be overt bleeding, either into the bed or into the superficial tissues, which is usually easily detected by ward staff. Alternatively, bleeding can be covert, usually into the retroperitoneal space. The latter possibility should always be considered if a patient is in unstable cardiovascular condition after TEVAR. If clinical signs are equivocal, urgent CT scanning should be used to diagnose retroperitoneal hematoma. Surgical exploration and repair are usually indicated, because significant arterial injury after passage of large delivery devices must excluded.

\section{SPINAL CORD PROTECTION}

The incidence of spinal cord injury after TEVAR is at least $3 \%$ and may be as high as $6 \% \cdot{ }^{8,9}$ Understanding spinal cord protection is vitally important for safe TEVAR and is an area of which cardiologists or peripheral vascular surgeons may have little knowledge. Recent evidence demonstrates that the extent of endograft coverage, presence of previous aortic reconstruction (proximal or distal to the TEVAR site), and patency of the left subclavian and hypogastric arteries may all be important in stratifying cord injury risk. ${ }^{9-11}$ Few endovascular centers have experience of intraoperative spinal cord monitoring, something we have recently called for in high-risk cases. ${ }^{12}$ We are able to draw on our own and others' experiences with methods to protect the spinal cord. ${ }^{13}$ The importance of cerebrospinal fluid drainage and management of the systemic arterial blood pressure is still poorly understood after TEVAR. Although this is common practice, it requires strict protocols to avoid error, which may have disastrous consequences. Other mechanisms that may be useful include reducing spinal cord metabolism during and immediately after intercostal and lumbar artery coverage. During TEVAR cases performed under general anesthesia, this may be an appropriate strategy, especially for patients at high risk for spinal cord injury.

\section{SUMMARY}

In the near future, TEVAR techniques may replace many of the indications for open surgery in the thoracic aortic segment. Clinicians with endovascular skills but without experience of thoracic aortic surgery should only undertake TEVAR as part of a team that includes a cardiovascular surgeon. Surgeons should be involved and should be aware of all techniques needed for TEVAR planning and stent placement. They should be involved in spinal cord risk assessment and protection and should be aware of both open and endovascular techniques that can be used to overcome the most common complications of TEVAR.

\section{References}

1. Dake MD, Miller DC, Semba CP, Mitchell RJ, Walker PJ, Liddell RP. Transluminal placement of endovascular stent-grafts for the treatment of descending thoracic aortic aneurysms. N Engl J Med. 1994;331:1729-34.

2. Makaroun MS, Dillavou ED, Kee ST, Sicard G, Chaikof E, Bavaria JE, et al. Endovascular treatment of thoracic aortic aneurysms: results of the phase II multicenter trial of the GORE TAG thoracic endoprosthesis. J Vasc Surg. 2005;41:1-9.

3. Fairman RM, Criado F, Farber M, Kwolek CJ, Mehta M, White R, et al. Pivotal results of the Medtronic Vascular Talent Thoracic Stent Graft System for patients with thoracic aortic disease: the VALOR trial. J Vasc Surg. 2008;48:546-54.

4. Matsumura JS, Cambria RP, Dake MD, Moore RD, Svensson LG, Snyder S. International controlled clinical trial of thoracic endovascular aneurysm repair with the Zenith TX2 endovascular graft: 1-year results. J Vasc Surg. 2008;47:247-57; discussion 257.

5. HESonline: Hospital Episode Statistics [Internet]. London: National Health Service. c2010-11 [cited]. Available from: http://www.hesonline.nhs.uk.

6. Kpodonu J, Rodriguez-Lopez JA, Ramaiah VG, Diethrich EB. "Cracking and paving": a novel technique to deliver a thoracic endograft despite ilio-femoral occlusive disease. J Card Surg. 2009;24:188-90.

7. Abu-Ghaida AM, Clair DG, Greenberg RK, Srivastava S, O'hara PJ, Ouriel K. Broadening the applicability of endovascular aneurysm repair: the use of iliac conduits. J Vasc Surg. 2002;36:111-7.

8. Cheng D, Martin J, Shennib H, Dunning J, Muneretto C, Schueler S, et al. Endovascular aortic repair versus open surgical repair for descending thoracic aortic disease: a systematic review and meta-analysis of comparative studies. J Am Coll Cardiol. 2010;55:986-1001.

9. Feezor RJ, Martin TD, Hess PJ Jr, Daniels MJ, Beaver TM, Klodell CT, et al. Extent of aortic coverage and incidence of spinal cord ischemia after thoracic endovascular aneurysm repair. Ann Thorac Surg. 2008;86:1809-14; discussion 1814. 
10. Matsuda H, Ogino H, Fukuda T, Iritani O, Sato S, Iba Y, et al. Multidisciplinary approach to prevent spinal cord ischemia after thoracic endovascular aneurysm repair for distal descending aorta. Ann Thorac Surg. 2010;90:561-5.

11. Riga CV, Bicknell CD, Cheshire NJ. Hybrid and endovascular therapy for extensive thoracoabdominal aortic disease. J Thorac Cardiovasc Surg. 2010;140(6 Suppl):S168-70; discussion S185-90.
12. Cheshire N. Debate: spinal cord monitoring is as essential for endovascular as for open surgical thoracic procedures - for the motion. Presented at: 34th Charing Cross International Symposium; 2012 Apr 16; London.

13. Bicknell CD, Riga CV, Wolfe JH. Prevention of paraplegia during thoracoabdominal aortic aneurysm repair. Eur J Vasc Endovasc Surg. 2009;37: $654-60$ 